\title{
An Autostereoscopic Display System for Image-Guided Surgery Using High-Quality Integral Videography with High Performance Computing
}

\author{
Hongen Liao ${ }^{1}$, Nobuhiko Hata ${ }^{1}$, Makoto Iwahara ${ }^{1}$, Ichiro Sakuma ${ }^{2}$, and \\ Takeyoshi Dohi ${ }^{1}$ \\ ${ }^{1}$ Graduate School of Information Science and Technology, The University of Tokyo \\ \{liao, noby, iwahara \} eatre.t.u-tokyo.ac.jp \\ ${ }^{2}$ Graduate School of Frontier Sciences, The University of Tokyo \\ \{sakuma, dohi\}@miki.pe.u-tokyo.ac.jp
}

\begin{abstract}
This paper presents a high-quality glass-less autostereoscopic display system that is integrated into image-guided surgery. The glass-less autostereoscopic image was created by employing a modification of Integral Videography (IV), which is an animated extension of Integral Photography. IV records and reproduces 3-D images using a micro convex lens array and plat display, which can display geometrically accurate 3-D autostereoscopic images and reproduce motion parallax without any need for special viewing devices. This paper reports the use of high-resolution IV display and high performance computing for producing medical 3-D image. We evaluate the feasibility of this display by using a set of medical image with segmentation and reproducing 3-D $\mathrm{CT}$ and MRI autostereoscopic image for surgical planning and intra-operative guidance. The main contribution of this paper is application and modification of medical 3-D display technique originally developed in high-resolution autostereoscopic display and high performance computing.
\end{abstract}

\section{Introduction}

Image-guided surgery has been evolved by incorporating advanced technology from medical imaging, computer science, sensor engineering and robotics [1-2]. The objective of the image-guided surgery is to enhance the surgeon's capability to utilize medical imagery to decrease the invasiveness of surgical procedures and increase their accuracy and safety. These systems focused on surgical guidance tend to present the surgeon with data that was gathered prior to surgery, track surgical instruments within the operating field, and render the tracked devices along with the data.

Stereoscopic technique has been taking an important roll in image assisted surgery and diagnosis recently, with various modes of visualization on offer [3-4]. Among previous reported methods use polarized or shuttering glasses originally developed in augmented reality domain and applied in surgical simulation and diagnosis. However, the binocular stereoscopic display reproduces the depth of projected objects by fixed binoculars, and produces a disparity as the images for left and right eye are prepared 
separately. Therefore, as is discussed in [5], this is always potential inconsistencies of depth perception among viewers. In the case of this kind of stereoscopic displays, they can observe pre-fixed 2-D images, which create quasi 3-D images with distortion. Subjectively they perceive it as 3-D, but there may be significant inaccuracies in registration. Motion parallax, an alternative stereoscopic vision, cannot be reproduced without wearing a tracking device.

Our previous reports proposed an approach to overcome these issues of binocular stereoscopic vision by using Integral Videography (IV), which was an animated extension of Integral Photography (IP) [6]. IV projects a computer-generated graphical object generated by multiple rays coming through micro convex lens array. The detailed description of IP and IV can be found in [7-9]. We further extended our work for surgical assistance in orthopedic surgery [9] and an intra-operative navigation in the trials of clinical application [7].

Though the advantage of IV has been proven in both feasibility studies and clinical applications [7-9], it is necessary to develop a high quality 3-D visualization system that integrates capabilities for data analysis and on-line interventional guidance into the setting of interventional image. The motivation of this paper is to overcome these issues by using multi-projection autostereoscopic IV display which combined with high performance computing (HPC) for fast rendering and data analysis software for pre-surgical / intra-operative data segmentation. We evaluate the feasibility of this display by developing a 3-D CT and MRI autostereoscopic image and applying for surgical planning and guidance.

\section{Materials and Methods}

A schematic presentation of the high-quality multi-projection IV display system is presented in Fig.1. The components of the display system include: (1) High-quality multi-projection IV autostereoscopic display system; (2) Image analysis and data segmentation of key anatomical and function structure; (3) High performance computing for parallel rendering in surgical autostereoscopic display.

\subsection{High-Quality IV Autostereoscopic Surgical Display}

The spatial resolution of the IV autostereoscopic image projected is proportional to the ratio of the lens diameter in the lens array to the pixel pitch of display. Thus, the projected pixel pitch needs to be made much smaller. In our system, multiple projectors of the display are arranged in display array, producing a high-resolution and high-density image across a rear projection screen by utilizing long zooms lens projection technique.

In this case, an important issue is the coordination of multiple commodity projectors to achieve seamless edge blending and precise alignment. Seamless edge blending can remove the visible discontinuities between adjacent projectors. We obtain precise alignment (or misalignment) information with a digital camera by measuring the position and color features of the pixels projected on a screen, and then feedback the calibration information to the image-processing hardware to make fine 
adjustments of the projected image on the screen. It requires only the coarsest physical alignment of the projectors.



Fig. 1. Schema of the high quality IV autostereoscopic surgical display

An UDP-based network software mechanism synchronized each frame of the video images. The video output from each PC was not directly supplied to projectors, but rather the video signal went through image processing hardware that worked as a filter to geometrically warp and modulate the color of the image encoded in the video signal.

By the nature of the IV principle described, the high resolution and high-density image projected on screen must be free from distortion and reflection, so an antireflective antistatic coating flat screen is used to display the image. This screen is placed in the rear of micro convex lens array. When the rendered elemental IV image is projected on the screen, the autostereoscopic image will be formatted to a spatial image.

\subsection{Image Analysis and Data Segmentation}

Data analysis software (3D Slicer, developed at the Artificial Intelligence Laboratory, Massachusetts Institute of Technology, Cambridge, Mass, and at Brigham and Women's Hospital, Harvard Medical School, Boston, Mass [10]) was installed on an independent workstation (SunBlade100; Sun Microsystems, Mountain View, CA) and connected with the CT scanner or MR imager by a network cable. This software was then customized to provide data analysis for image-guided planning and navigation. A server program that continuously sends the acquired CT or MR images and information on the position was integrated with the CT/MR system. High-resolution three-dimensional CT/MR data were acquired just before the surgical procedures or during the operation and were input on the computer with the data analysis software. 
Volumetric data can be semi-automatically segmented using the 3D Slicer's suite of editing tools. One strength of our system is that effects can be visualized by overlaying the edited volume translucently on the original volume and exploring both in the three-dimensional view, as shown in Fig.2. The segmentation data and the image analysis results are transmitted to high performance computer.

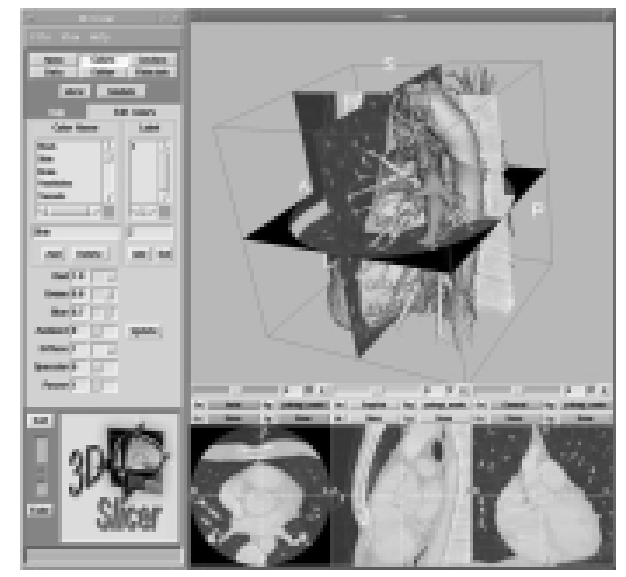

Fig. 2. The output of segmentation is displayed with variable opacity.

\subsection{High Performance Computing and Parallel Display}

We implemented the parallelized IV rendering software in SMP-based high performance computer. We also developed a distributed system to separate the IV display unit from HPC unit since the size of HPC unit is not suitable to fit in operative room. The communication to transform the rendered image to the display unit is possible by using TCP/IP Client-Server mechanism combined with socket communication. A Tcl/tk application works on the display as socket client.

The overall strategy in increasing the rendering performance of IV in this study is to use parallel computing integrated in shared memory HPC. The basic principle of IV rendering is projecting rays through a lens originated from the sub-division of computer display placed in the backside of lens array. The rendering is computationally almost equivalent to ray-tracing in volume rendering.

We integrated Message Passing Interface (MPI) [11] to increase the rendering performance of IV. MPI is an industry-wide message-passing standard common for programming in a distributed or a shared memory environment [12]. The parallelization is based on static partitioning of the rendering image. Each node in a cluster computer is assigned a section of the line to reconstruct. The node elaborates their section in parallel and, once finished, sends reconstructed section to a master process. The master process is in charge of $\mathrm{I} / \mathrm{O}$, or network communication API, which collects source images and sends the rendered image to parallel display cluster. 


\subsection{Clinical Setting of IV Display System}

Since the large device is limited in the operative room, we put the imaging units of multi-projection device for displaying IV images in the operation room, and high performance computer out of the operative room.

\section{Clinical Feasibility Studies}

To evaluate the feasibility of this study, we applied this display system to imageguided surgery. These autostereoscopic images can enhance the surgeon's capability to utilize medical 3-D imagery to decrease the invasiveness of surgical procedures and increase their accuracy and safety. These applications include surgical planning, surgical guidance, and surgical guidance with intra-operative updates. Our prior works focused on surgical guidance to present the surgeon with IV image that was gathered in pre-operative, track surgical instruments within the operating field and display in a real-time IV autostereoscopic image [7]. In this study, we perform surgical planning and simulation by this newly developed IV autostereoscopic display.

\subsection{Equipments and Results}

We developed a multi-projector system with 9 projectors (XGA, U2-1110, PLUS Vision Corp., Tokyo Japan), arranged in a $3 \times 3$ array, which producing a display of $2868 \times 2150$ pixels across a $241 \times 181 \mathrm{~mm}(302.4 \mathrm{dpi})$ rear projection screen by utilizing long zoom lens projection technique. The lenses of long zoom lens projection using in this projector include 8 sets and 12 pieces of lenses. The pitch of each pixel projected on the screen is $0.084 \mathrm{~mm}$. Micro lens array placed on the screen has hexagonal micro lenses with diameter of approximately $1.008 \mathrm{~mm}$, covers 12 pixels in the long zoom lens projection screen. We obtain precise alignment information with a camera (Nikon D1X digital camera) and transmit the correction parameter to the imageprocessing hardware (Model PA99, XGA, 65MHz, 60 frames/ second, System Development Laboratory, Hitachi, Ltd).

In order to observe the 3-D image with correct motion parallax, one should observe at a distance of about $50 \mathrm{~cm}$ from the system. The condition wherein the projected IV image was continuously observed was examined (Fig.3). The IV autostereoscopic display has a spatial resolution of $1 \mathrm{~mm}, 2 \mathrm{~mm}, 3 \mathrm{~mm}$ in $25 \mathrm{~mm}, 45 \mathrm{~mm}, 60 \mathrm{~mm}$ front and behind the lens array separately.

\subsection{High Performance Computing}

Each node in a cluster computer is assigned a section of the line to reconstruct. Trying on different CPUs from 1 to 60 , each trial are repeated 100 times. The results from the performance test indicated that the scalability is guaranteed in HPC (SunFire 15K, UltraSparc III $900 \mathrm{MHz} \times 60 \mathrm{CPU}, 60 \mathrm{GByte}$ RAM, Mountain View, CA) with up to 48 nodes, and the additional nodes will not increase computing speed. The computing time has decreased from 40 seconds/frame (XGA, CPU: Pentium III $800 \mathrm{MHz}$, 
Memory: $512 \mathrm{MB}$ DIMM) to 0.2 seconds/frame with HPC. The feasibility study indicated that the rendering performance achieved with the proposed method is about 200 -times as fast as previous system, which is satisfactory and suitable in surgical setting.

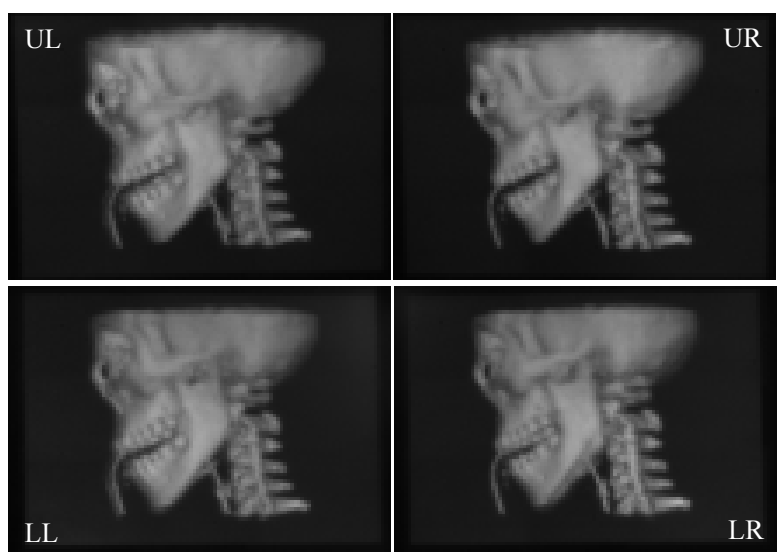

Fig. 3. Motion parallax of IV autostereoscopic skull images, taken from various directions. The letter denotes the position of the observer to the IV autostereoscopic image: UL: upper left, UR: upper right, LL: lower left, LR:lower right .

\subsection{Pre-surgical Planning and Intra-operative Guidance}

Intra-operatively, IV autostereoscopic image can help with the navigation of instruments by providing a broader view of the operation field. In combination with robotic and surgical instrument, it even can supply guidance by pre-defining the path of a biopsy needle or by preventing the surgical instruments from moving into critical regions.

We evaluated the usefulness of the newly developed system in operative setting. In clinical feasibility study, we performed CT scanning to take photo of in-vivo knee. The volumetric CT images of knee $(512 \times 512$ pixels $\times 347$ slices, thickness of $0.5 \mathrm{~mm})$ were segmented by 3D Slicer and the results were rendered by HPC and displayed in multi-projection IV display. We integrated IV segmentation image in knee arthroplasty (Fig.4a), and superimposed IV image into the patient in surgical implementation. These combinations enabled safe, easy, and accurate navigation.

We also performed CT scanning to take photo of in-vivo human heart 10 phases in one cardiac cycle. The volumetric CT images of human heart $(512 \times 512$ pixelsc $\times 180$ slices for one phase, thickness of $1.0 \mathrm{~mm}$ ) were rendered 10 times separately in one heartbeat. The rendered elemental IV images were projected continually on the IV stereoscopic display with the same heartbeat period of the patient (Fig.5b). 


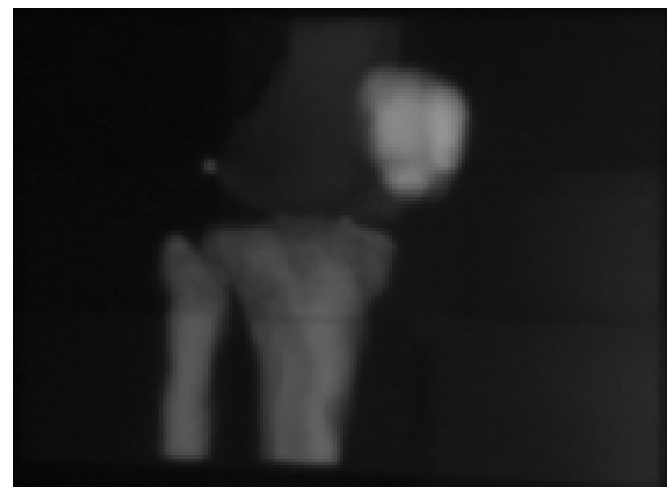

Fig. 4. IV image for surgical planning and navigation: Surgical implementation integrated with IV image for knee arthroplasty with data segmentation.
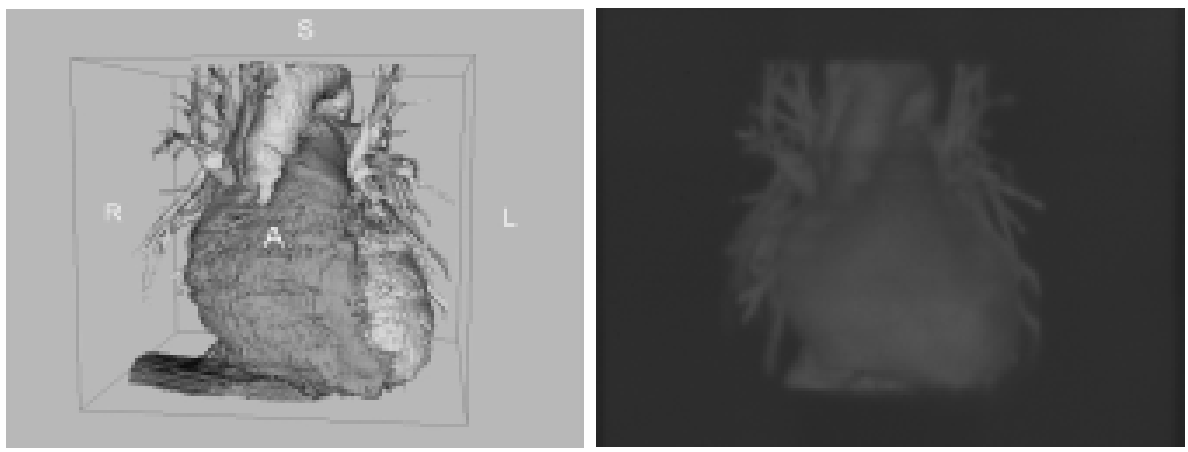

Fig. 5. IV CT autostereoscopic animated image: The heart beat of patient has a rate of 63 beats per minute, cardiac cycle of $0.95 \mathrm{~s}$. (a) Segmentation data by 3 -D Slicer. (b) IV image by multiprojection IV display.

\section{Discussions and Conclusion}

The IV autostereoscopic display system developed in this study has three primary merits: Superimposing the real, intuitive 3-D image for medical diagnosis and surgical guidance; Avoiding the need of extra devices such as the wearing of special glasses, and offering a geometrical accuracy image over the projected objects (esp. Depth perspective); Visibility of motion parallax over a wide area, simultaneous observation by many people.

As accuracy is most important element in medical imaging, this system has advantages over other 3-D image display method. Theoretically, when the ideal lens is utilized, IV can provide a three-dimensional display that is free from any discontinuous change of images that occur with the observer's movement and with the same resolution that conventional two-dimensional displays feature [13]. The measurements of spatial resolution of IV autostereoscopic display show the quality of 
IV image can be improved. Actually, the IV autostereoscopic display systems developed in this study have a pixel density of only about 300dpi. The spatial resolution of the 3-D image projected is proportional to the ratio of the lens diameter in the lens array to the pixel pitch of display. Thus, both the lens diameter and the projected pixel pitch need to be made much smaller. One of the possible solutions to realize higher pixel density is the use of more multi-projectors or projector with higher resolution (SXGA or more pixels) to create more high-resolution image. A more large computation power is also needed to correspond to multi-projector system.

The HPC using for the IV image rendering can accelerate calculation. The results of the performance test indicated that the scalability is guaranteed in HPC with up to 48 nodes. This may be partially due to the network traffic jam, yet primary due to unorganized communication of MPI. Additional performance analysis is required to fully investigate cause of the unbalanced scalability.

In conclusion, we have developed a high resolution with high pixel density device for IV autostereoscopic display system using multi-projector and reduction projection method. The feasibility study indicated that the multi-projector and parallel rendering performance achieved with the proposed method is satisfactory and suitable in surgical diagnosis and planning setting. We evaluate the feasibility of this display by developing a 3-D CT autostereoscopic image and applying it to surgical planning. The main contribution of this paper is application and modification of autostereoscopic surgical display technique originally developed in high-resolution multi-projector autostereoscopic display system and high performance computing.

Acknowledgements. This study was partly supported by the Grant-in-Aid for the Development of Innovative Technology (12113) by the MEXT in Japan. We are deeply indebted to Ferenc A. Jolesz and Ron Kikinis of Brigham and Women's Hospital, Boston, Mass, for donating the 3D Slicer software, and Takafumi Koike, Yasuyuki Momoi, Masami Yamasaki, Haruo Takeda of the System Development Laboratory, Hitachi, Ltd, for their contribution in seamless technique in multiprojector display.

\section{References}

1. A.F.Hinsche, R.M.Smith: "Image-guided surgery," Current Orthopaedics, Vol.15, No.4, pp.296-303, 2001.

2. Aaron Fenster, Kathleen Surrya, Wendy Smitha, Jeremy Gilla and Dónal B. Downey: "3D ultrasound imaging: applications in image-guided therapy and biopsy," Computer \& Graphics, Vol.26, No.4, pp.557-568, 2002.

3. M.A.Guttman, E.R.McVeigh, "Techniques for fast stereoscopic MRI," Magnetic Resonance in Medicines, Vol.46, pp317-323, 2001.

4. M.Blackwell, C.Nikou, A.M.Digioia, T.Kanade, "An image overlay system for medical data visualization," Medical Image Analysis, Vol.4 pp.67-72, 2000.

5. B.T.Backus, et al., "Horizontal and vertical disparity, eye position, and stereoscopic slant perception,” Vision Research, Vol.39 No.6, pp.1143-1170, 1999.

6. M.G.Lippmann, "Epreuves reversibles donnant la sensation du relief," J. de Phys Vol.7, 4th series, pp821-825, 1908 . 
7. H.Liao, S.Nakajima, M.Iwahara, E.Kobayashi, I.Sakuma, N.Yahagi, T.Dohi, "Intraoperative Real-Time 3-D Information Display System based on Integral Videography," Medical Image Computing and Computer assisted Intervention MICCAI2001, LNCS 2208, pp.392-400, 2001.

8. Y.Masutani et al., "Development of integral photography-based enhanced reality visualization system for surgical support," Proc. of ISCAS'95, pp16-17, 1995.

9. S.Nakajima, K.Nakamura, K.Masamune, I.Sakuma, T.Dohi, "Three-dimensional medical display with computer-generated integral photography," Computerized Medical Imaging and Graphics, 25, pp235-241, 2001.

10. Jolesz FA, Nabavi A, Kikinis R. Integration of interventional MRI with computer-assisted surgery. J Magn Reson Imaging, Vol.13, pp.69-77, 2001.

11. Peter S.Pacheco, "Parallel Programming with MPI," Morgan Kaufmann, 1996

12. W.S.Lin, R.W.H.Lau, K.Hwang, X. Lin, Y.S.Cheung, "Adaptive Parallel Rendering on Multiprocessors and Workstation Clusters", IEEE Trans. Parallel and Distributed Systems, Vol.12, No.3, pp.241-258, 2001.

13. H.Hoshino, F.Okano, H.Isono, I.Yuyama, "Analysis of resolution limitation of integral photography,” Optical Society of America, Vol.15, No.8, pp2059-2065, 1998. 